\title{
Heimlicher Hochdruck bei Typ-1-Diabetikern
}

\author{
Diabetiker mit Hypertonie \\ haben ein hohes Risiko für \\ Nephropathie und Reti- \\ nopathie. Sonderformen \\ der Hypertonie, nämlich \\ die maskierte Hypertonie \\ und nächtliche Blutdruck- \\ steigerungen, werden bei \\ Blutdruckmessungen in \\ Praxis und Klinik überse- \\ hen, könnten aber bei Dia- \\ betikern ernsthafte Folgen \\ haben.
}

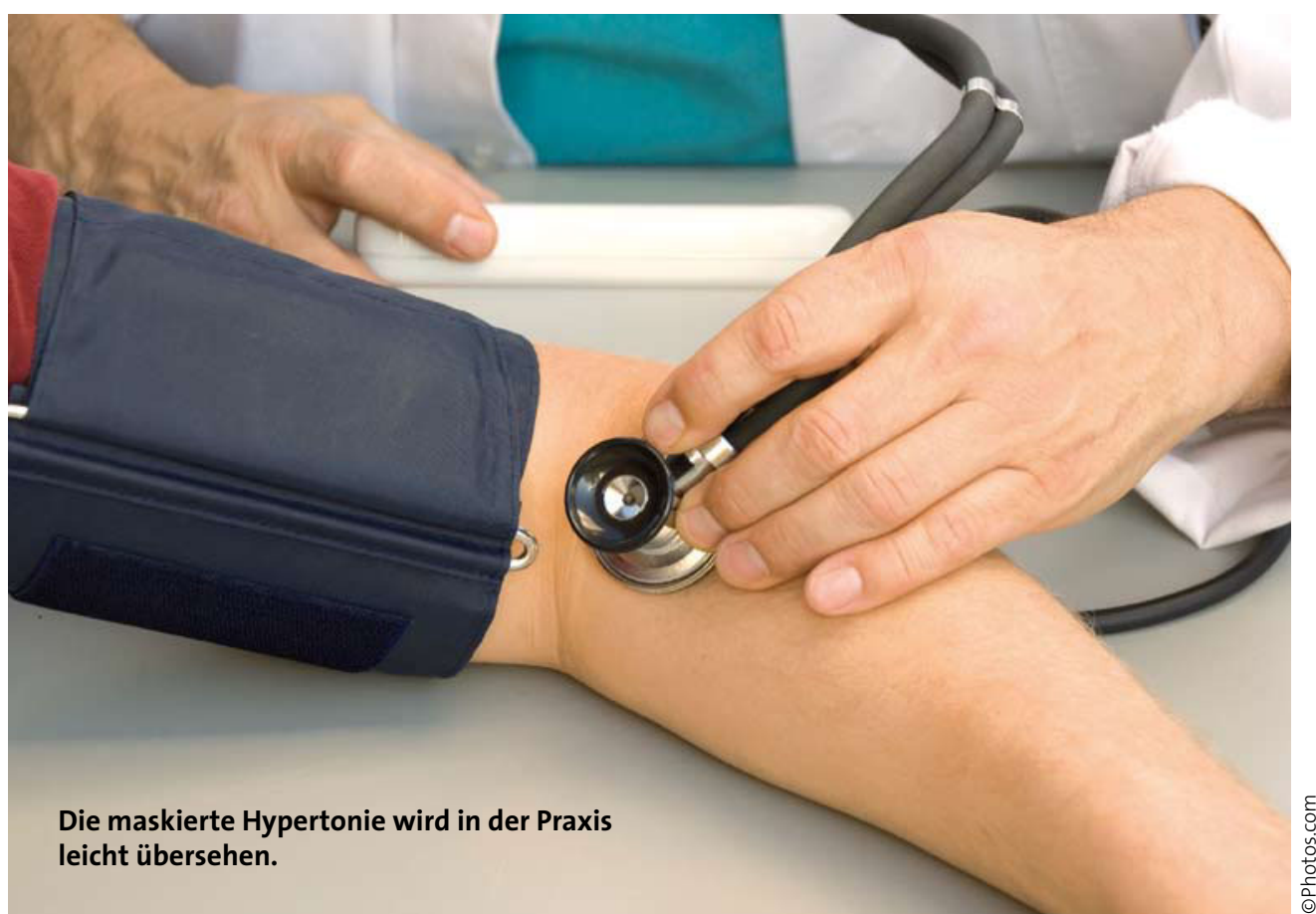

- Bei 188 Typ-1-Diabetikern eines Zentrums wurde der Blutdruck konventionell in der Ambulanz und durch ambulante Langzeitmessung (ABDM) bestimmt. Als maskierte Hypertonie wurden normaler Blutdruck in der Ambulanz $(<130 / 80 \mathrm{mmHg})$ und erhöhter Blutdruck bei der ABDM (Tagesblutdruck 135/85 mmHg oder höher) ohne Einnahme von Antihypertensiva klassifiziert.

Eine maskierte nächtliche Hypertonie war definiert als Normotonie in der Ambulanz $(<130 / 80 \mathrm{mmHg})$ mit erhöhten ABDM-Werten in der Nacht $(\geq 120 / 70 \mathrm{mmHg}$ ). Der Augenhintergrund wurde auf Retinopathie hin untersucht und die Eiweißausscheidung im Urin als Kriterium für eine Nephropathie (> $20 \mu \mathrm{g} / \mathrm{min})$ gemessen.

Ergebnisse: Von 188 Diabetikern (mittleres Alter 32 Jahre) waren in der Ambulanz 103 normoton, 66 hyperton und 19 hatten eine Praxishypertonie. Von den 103 „normotonen“ Patienten hatten $14(13,6 \%)$ bei der Langzeitmessung einen erhöhten Blutdruck, d.h. eine maskierte Hypertonie, und 24 $(23,3 \%)$ einen erhöhten Blutdruck in

der Nacht, d.h. eine nächtliche, maskierte Hypertonie. Letztere hatten signifikant häufiger eine diabetische Retinopathie als solche mit normalem Blutdruck in der Ambulanz und bei der ABDM.

\section{Kommentar}

Nicht diabetische Patienten mit maskierter Hypertonie (auch Praxisnormotonie oder Out-of-Office-Hypertension genannt) haben ein erhöhtes kardiovaskuläres Risiko. Diese Untersuchung bestätigt an einem relativ kleinen Kollektiv, dass es die maskierte Hypertonie am Tag und in der Nacht auch bei Typ-1-Diabetikern gibt und dass die nächtliche Hypertonie die diabetische Retinopathie begünstigt. Bei größerer Patientenzahl und längerer Beobachtungsdauer würde sicher auch ein erhöhtes Risiko für diabetische Nephropathie und kardiovaskuläre Komplikationen entdeckt werden. Außerdem besteht kein vernünftiger Zweifel, dass auch Typ-2-Diabetiker von maskierter Hypertonie betroffen und von ihren Folgen bedroht sind.
Typ-1-Diabetiker mit normalem Blutdruck in der Ambulanz haben häufig eine Hypertonie im Alltag und einen zu hohen Blutdruck in der Nacht. Die nächtliche Hypertonie geht mit einem erhöhten Retinopathierisiko einher. wa jedem fünften Diabetiker. Sie wird durch die Blutdruckmessung in Praxis und Klinik nicht erkannt, beinhaltet aber ein erhöhtes Risiko für diabetische mikro- und makrovaskuläre Komplikationen. Deshalb sollte bei jedem Diabetiker mit normalem Praxisblutdruck vorsichtshalber eine Langzeitmessung, ersatzweise eine Selbstmessung über zwei Wochen veranlasst werden.

H. HolzG REVE

- T. C. Rodrigues et al.

Masked hypertension, nocturnal blood pressure and retinopathy in normotensive patients with type 1 diabetes. Diabetes Res. Clin. Pract. 87 (2010) 240-245
Eine maskierte Hypertonie besteht bei et- 